\title{
Conservative management of gigantic splenic cyst during pregnancy; a differential diagnosis for chest pain caused by the forgotten organ
}

\author{
Montasser A. Mahran • Roger Bodley • \\ Marwan Farouk • Felicity Ashworth
}

Received: 21 September 2008 / Accepted: 27 November 2008 / Published online: 10 January 2009

(C) Springer-Verlag 2008

\begin{abstract}
Splenic cyst is extremely rare in pregnancy. All the six cases that had been described in literatures were treated surgically. However, we report the first case of a huge splenic cyst during pregnancy managed by conservative approach in the form of analgesia, antibiotics and percutaneous aspiration.
\end{abstract}

Keywords Pregnancy $\cdot$ Spleen $\cdot$ Cyst $\cdot$ Haemorrhage

\section{Case report}

A 34-year-old woman presented in her second pregnancy at 24 weeks gestation with a 4-week history of increasing left upper abdominal and left shoulder pain. There was no history of abdominal trauma or foreign travel.

She was admitted to the hospital to investigate the pain, particularly to exclude a pulmonary embolism and computed tomography pulmonary angiography (CTPA) was performed. This excluded a pulmonary embolism, but revealed a large splenic cyst. Palpation of the abdomen showed an enlarged spleen. The diagnosis was then confirmed by abdominal ultrasound scan, which revealed a huge round $20 \mathrm{~cm}$ splenic cyst with a smooth thin wall, filled by 21 of homogenous fluid with no signs of malignancy (Fig. 1). The serology for hydatid IgG was negative. Platelets, liver and kidney function tests were

M. A. Mahran

Obstetrics and Gynaecology, Ipswich Hospital,

Suffolk, UK

R. Bodley $\cdot$ M. Farouk $\cdot$ F. Ashworth $(\bowtie)$

Stoke Mandeville Hospital,

Bucks HP21 8AL, UK

e-mail: Felicity.Ashworth@buckshosp.nhs.uk normal. However, the haemoglobin level (Hb) on admission was $8.3 \mathrm{~g} / \mathrm{dl}$, a reduction of $1.3 \mathrm{~g} / \mathrm{dl}$ compared to the previous week. This raised the possibility of a haemorrhage into the cyst. However, the ultrasound didn't suggest this.

In liaison with the surgical and radiology teams, it was decided to postpone aspiration of the cyst until a time of fetal maturation, to avoid unwarranted effect on the fetus should laparotomy be needed. Pain was controlled by morphine and pethidine. Iron tablets were given for anaemia and a daily full blood count revealed no further drop in the $\mathrm{Hb}$ level. Two units of cross-matched blood were made available for any emergency.

At 26 weeks the patient experienced shivering and a fever of $39^{\circ} \mathrm{C}$. Haemoglobin dropped to $7.8 \mathrm{~g} / \mathrm{dl}$ with raised C-reactive protein of $99 \mathrm{mg} / \mathrm{l}$. Two units of blood were transfused and intravenous co-amoxiclav was started to treat the septicaemia which was presumed to be from the infected haemorrhagic cyst. Betamethasone was also given in case of preterm delivery. Abdominal ultrasound showed no increase in the size of the cyst and satisfactory fetal growth. The patient's general condition improved with the treatment.

At 27 weeks a percutaneous aspiration under ultrasound guidance was performed. This was earlier than planned to avoid further episodes of infection. Three and a half litres of greyish brown fluid were aspirated and coagulase negative staphylococci sensitive to co-amoxiclav were cultured. The patient showed a good improvement and was discharged 2 days later on oral co-amoxiclav.

Ultrasound scans at 32 and 35 weeks revealed reaccumulation of the cyst to measure $15 \mathrm{~cm}$. No further aspiration was performed, as the patient was asymptomatic. At 40 weeks the patient went into spontaneous labour and had a normal vaginal delivery. Ultrasound scan 6 weeks following the delivery showed persistence of the cyst. This 


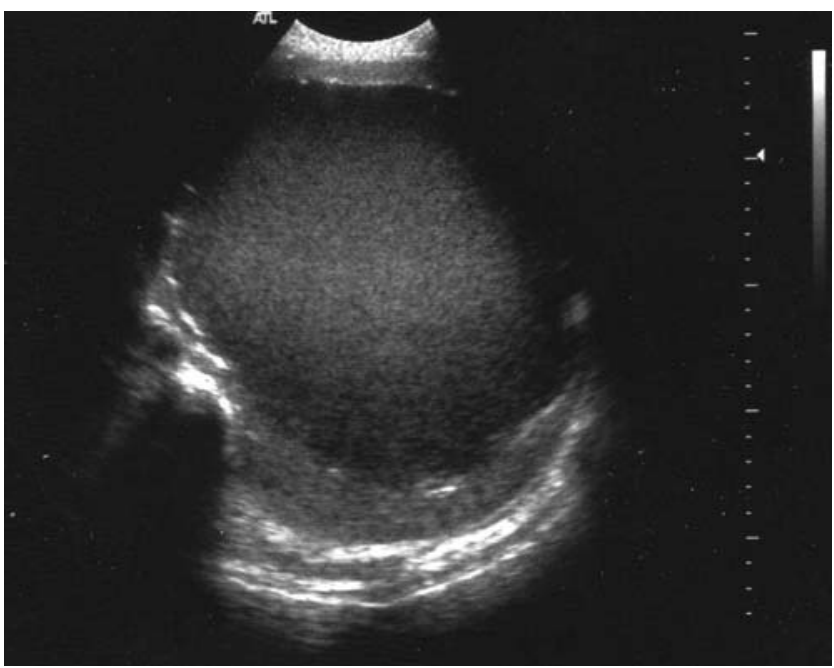

Fig. 1 The abdominal ultrasound showing the huge splenic cyst

was treated with re-aspiration and microbiology showed no growth on culture. One year later, the patient had her third pregnancy and antenatal and post-natal ultrasound scans confirmed complete disappearance of the cyst.

\section{Discussion}

Splenic cyst is extremely rare in pregnancy. Six cases have been described in the literature and all were treated surgically. We report the first case of successful conservative approach of huge splenic cyst in pregnancy.

Splenic cysts are classified into primary and secondary. The primary causes are parasitic, congenital and neoplastic. Secondary cysts may develop secondary to trauma, haemorrhage, infarction-degeneration and inflammation. In pregnancy, haematoma following abdominal trauma is the commonest cause for secondary cysts, as splenic infarction resulting from hormonal changes of pregnancy increases the vulnerability of this organ and thus accounts for high incidence of secondary splenic cyst [1].

Splenic cyst carries the risks of infection, haemorrhage and rupture. Because it is extremely rare in pregnancy, it is an unusual differential diagnosis for left upper quadrant abdominal pain and chest pain in pregnancy. Spleen rupture during pregnancy is known as splenic emergency syndrome which is characterised by the severe onset of pain in the left hypochondrium or epigastrium, followed by haemorrhagic shock [2]. However, the true incidence of splenic cyst rupture during pregnancy is unknown as only six cases of splenic cyst with pregnancy have been described in the literature [3].

Splenic rupture has been recorded in pregnancy mainly in the third trimester. This has been attributed to the hypervolaemic state, splenic enlargement and diminished peritoneal cavity volume, due to the enlarged uterus and muscular contractions during labour [4]. With such a serious event, there is an increased risk of fetal death. High maternal mortality rates have been reported (up to 70\%) [2].

The majority of splenic cysts are asymptomatic. However, distension of the splenic capsule with a huge cyst will cause left shoulder pain, upper left quadrant pain or chest pain. This may be diagnosed initially as pulmonary embolism as in our case. Pressure on the left kidney may cause proteinuria or even hypertension in pregnancy. This may lead to an erroneous diagnosis of pre-eclampsia especially if associated with thrombocytopenia due to the circulatory changes in the spleen [3]. For this reason it is essential to perform a full abdominal examination to reach the correct diagnosis for this unusual clinical condition. Our diagnosis of the splenic cyst was made after a CTPA and was missed on initial examination as nowadays the spleen seems to be 'the forgotten organ'.

Serology to rule out parasitic aetiology is essential. Rupture of a hydatid cyst could result in anaphylactic shock. Imaging using ultrasound, computed tomography or magnetic resonance imaging (MRI) will help to determine the size of the cyst and it's relation to surrounding structures in case surgery is needed. Imaging can also help in excluding malignancy [5]. This patient was assumed to have a primary congenital cyst. Other types had been excluded by history and laboratory findings.

It has been suggested that surgery should be the ideal treatment of splenic cyst if it is more than $5 \mathrm{~cm}$ in diameter, to reduce the risk of complications [6]. All the six cases of splenic cysts in pregnancy, described in the literature, were treated surgically either by laparotomy or laparoscopy, with or without splenectomy. Ideally, the splenic cyst should be treated in the second trimester to avoid the increased risk of splenic rupture in the third trimester or puerperium [3].

The decision to defer the aspiration, in case it led to surgery, was made because of the patient's presentation at 24 weeks, with the baby at borderline of viability. The diagnosis and treatment of any medical condition requiring surgery in pregnancy often raises questions about the safety of anaesthesia and surgery in these patients [7]. Splenectomy during pregnancy is regarded as a high-risk procedure for the mother and the fetus. It carries the risks of infection, thrombosis and bleeding. A maternal mortality rate of $10 \%$ and a high neonatal mortality rate due to premature labour have been reported after surgery [8]. These risks had been taken into consideration in our management.

When imaging studies suggest a benign cyst, aspiration is extremely useful. We present the first case of a large splenic cyst in pregnancy successfully treated with aspiration and preservation of the spleen to avoid the surgical and anaesthetic risks. Aspiration is a straightforward procedure and is considered to be safer than surgery as it is done 
under local anaesthesia with ultrasound guidance, even as an out patient procedure. However, it can increase the risk of adhesions and makes the splenectomy more difficult if needed in the future. Aspiration is not a suitable management for the malignant or hydatid cyst or for the recurrent symptomatic cyst after aspiration. Perhaps this can explain why the previous cases were managed surgically.

Percutaneous fluid aspiration can be performed for therapeutic purposes in symptomatic patients and in our case the aspiration was advised because of the suspected haemorrhage and infection in the cyst. More commonly, however, fluid aspiration is performed for diagnostic purposes, usually to differentiate between a simple splenic cyst and a splenic abscess. In non-pregnant patients aspirations of splenic cysts have been successful [9]. However, reaccumulation of the fluid has been reported following successful aspiration in pregnancy which necessitated surgery to remove the cyst [3]. Further studies are needed to find out if hypervolaemic changes of pregnancy will increase the chance of re-accumulation of the cyst or not. Indeed, it is essential to follow up the cyst by serial ultrasound to determine the need for further aspiration especially if the cyst is symptomatic.

A conservative approach in the form of analgesia, antibiotics and percutaneous aspiration has been shown to be effective in our case. Indeed this had prevented surgical intervention during pregnancy with preservation of the spleen. This case also illustrates that an abdominal problem can present with chest pain.

\section{References}

1. Triedman LJ, Raphael SI (1967) Nonparasitic cysts of the spleen: case report and review of the literature. Rare disorder, here associated with pregnancy, is cured by splenectomy. R I Med J 50(4):265-267

2. Tanchev S, Papova M, Slavov I (1992) The "splenic emergency syndrome" during pregnancy. A report of two cases. Akush Ginekol 31:32-34

3. Rotas M, Ossowski R, Lutchman G, Levgur M (2007) Pregnancy complicated with a giant splenic cyst: a case report and review of the literature. Arch Gynecol Obstet 275(4):301-305, Epub 2006 Aug 26

4. Gilbert CRA, Goldzieher JW, Cook TA (1964) Insidious rupture of the spleen or splenic vessels associated with pregnancy. J Abdom Surg 6:48-57

5. Wan YL, Cheung YC, Lui KW, Tseng JH, Lee TY (2000) Ultrasonographic findings and differentiation of benign and malignant focal splenic lesions. Postgrad Med J 76(898):488-493

6. Labruzzo C, Haritopoulos KN, El Tayar AR, Hakim NS (2002) Posttraumatic cyst of the spleen: a case report and review of the literature. Int Surg 87(3):152-156

7. Kuczkowski KM (2006) The safety of anaesthetics in pregnant women. Expert Opin Drug Saf 5(2):251-264

8. Burrows R (1999) Splenectomy during pregnancy: an option in treatment of autoimmune thrombocytopenic purpura. Br J Obstet Gynaecol 106(12):1330-1331

9. Lucey BC, Boland GW, Maher MM, Hahn PF, Gervais DA, Mueller PR (2002) Percutaneous nonvascular splenic intervention: a 10-year review. AJR Am J Roentgenol 179(6):1591-1596 